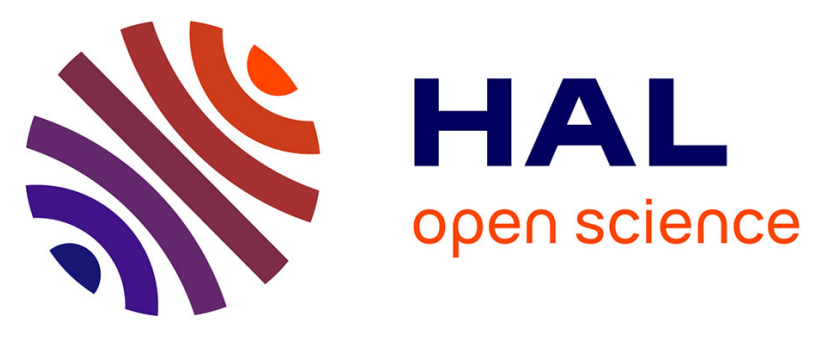

\title{
Digital Control of Residual Amplitude Modulation for Ultra-Stable Optical Cavity
}

Santerelli Falzon Tetsing Talla, Jacques Millo, Severine Denis, Clément Lacroute, Gwenhaël Goavec-Merou, Enrico Rubiola, Yann Kersalé

\section{To cite this version:}

Santerelli Falzon Tetsing Talla, Jacques Millo, Severine Denis, Clément Lacroute, Gwenhaël GoavecMerou, et al.. Digital Control of Residual Amplitude Modulation for Ultra-Stable Optical Cavity. International Frequency Control Symposium \& International Symposium on Applications of Ferroelectrics, Jul 2020, Keystone, United States. hal-03053011

\section{HAL Id: hal-03053011 \\ https://hal.science/hal-03053011}

Submitted on 10 Dec 2020

HAL is a multi-disciplinary open access archive for the deposit and dissemination of scientific research documents, whether they are published or not. The documents may come from teaching and research institutions in France or abroad, or from public or private research centers.
L'archive ouverte pluridisciplinaire HAL, est destinée au dépôt et à la diffusion de documents scientifiques de niveau recherche, publiés ou non, émanant des établissements d'enseignement et de recherche français ou étrangers, des laboratoires publics ou privés. 


\title{
Digital Control of Residual Amplitude Modulation for Ultra-Stable Optical Cavity
}

\author{
S. F. Tetsing Talla, J. Millo, S. Denis, C. Lacroûte, G. Goavec-Merou, E. Rubiola, Y. Kersalé \\ FEMTO-ST Institute, Université Bourgogne Franche-Comté, CNRS \\ ENSMM, 26 Rue de l'Épitaphe, 25030 Besançon, France \\ santerelli.tetsing@femto-st.fr; jacques.millo@femto-st.fr
}

\begin{abstract}
In this work, we present a technique to control and reduce the residual amplitude modulation (RAM) in the cavitystabilized laser, which is one of the main limitations of these systems. The RAM rises from polarization mismatch between electro-optic modulator (EOM) axis and polarizers implemented for the generation of the error signal by the Pound-Drever-Hall technique. Using digital control system, we have developed a simple and robust technique for an active reduction of the RAM acting on the temperature of the EOM in addition with a DC and high voltage applied accross the EOM crystal. We observe a reduction of the RAM to $0.25 \mathrm{ppm}$ compatible with a fractional frequency stability in the low $1 e^{-16}$ around $10 \mathrm{~s}$.
\end{abstract}

\section{INTRODUCTION}

Nowadays, the most stable and accurate atomic clocks are based on optical transitions [1]. In these clocks, the local oscillator is an ultra-stable laser (USL) stabilize to the so called clock transition frequency [2]. Thanks to outstanding fractional frequency stability demonstrated by these USL, $\sim 10^{-16}$ or lower for short integration times [3], they are also used for others applications, such as interferometric measurements [4], high-resolution spectroscopies [5], fundamental physics tests [6] and the generation of microwave signals using an optical frequency comb [7]. In practice, cavity stabilized laser is made of continuous laser whose frequency is locked to a resonance mode frequency of a high finesse Fabry-Perot cavity. The laser to cavity frequency detuning requiered for the stabilization, is measured using the Pound-Drever-Hall (PDH) technique [8] that need to imprint a sinusoidal phase modulation on the laser light. The imperfection of this phase modulation setup generates unwanted RAM that shift the measurement of the frequency detuning.

\section{Silicon FABRy-PERot CAVity}

The spacer and the mirrors substrate of the ultra-stable optical cavity are made from single crystal silicon material with the [111] direction along the optical axis. The cylindrical spacer with a length of $140 \mathrm{~mm}$ and a diameter of $100 \mathrm{~mm}$ is tapered on both end to reduce vibration sensitivity. Figure 1 shows the silicon cavity in it support with the optical axis oriented horizontally. This optical resonator is hold by three contact points in the vertical middle plane (orthogonal to the optical axis) and aligned to the crystalline orientation. The top contact point is provided by a mass of approximately $1 \mathrm{~kg}$ to obtain a force applied independent of the wide temperature range supported by the system. The dielectric amorphous coating of the two mirrors gives a finesse about 78000 for $\mathrm{TEM}_{00}$ mode around $1542 \mathrm{~nm}$. This low value is attributed to a poor polishing quality of the substrates. To take advantage of the strong mechanical quality factor of this cavity, it is cooled to $18.1 \mathrm{~K}$, which corresponds to one of its zero thermal expansion coefficients. The cryostat used to reached this temperature is based on a closed cycle heliumbased cooling machine (pulsed tube cryocooler, Cryomech).

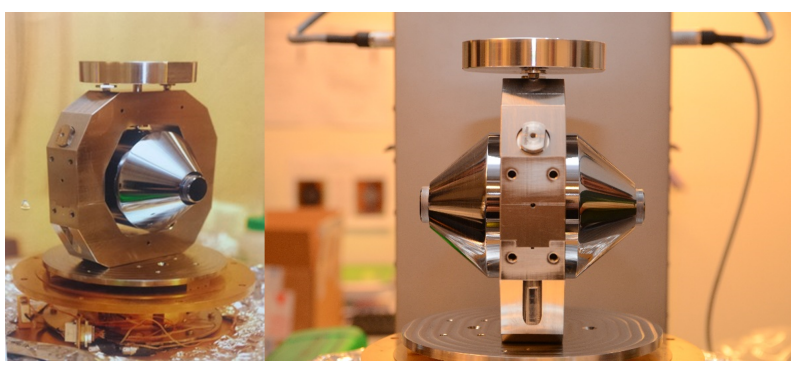

Fig. 1. Silicon Fabry-Perot cavity using in the cavity-stabilized laser.

\section{Residual Amplitude Modulation}

The PDH technique takes advantage of the large phase variation of the reflection coefficient function of the cavity as the frequency detuning. The phase modulation at frequency $\mathrm{f}_{m}$ generates two sidebands and a carrier. When the laser frequency is close to the cavity resonance, the carrier is partially reflected accumulating the phase of the cavity while the sidebands are reflected with a constant phase-shift. Interferences of these three spectral components on a fast photodiode and demodulation at $\mathrm{f}_{m}$ gives a reading of the phase and thus an error signal. The RAM is at frequency $\mathrm{f}_{m}$ and thus the photodiode provides a signal at $\mathrm{f}_{m}$ without any impact of the detuning.

By adding amplitude modulation to the model describing the generation of the PDH error signal [8], one calculate the following relation that shows the contribution of the RAM on the error signal:

$$
\varepsilon(t)=\frac{-8 \sqrt{P_{c} P_{s}}}{\Delta \nu_{c}} \delta \nu(t)+m P_{c}\left[\frac{P_{s}}{P_{c}}-\operatorname{Re}(R)\left(1+\frac{2 P_{s}}{P_{c}}\right)\right]
$$

where $P_{c}$ and $P_{s}$ are respectively the RF power in the carrier and sidebands; $\Delta \nu_{c}$ is the full width at half maximum of the optical cavity; $\delta \nu$ represents the frequency detuning between 
the laser and the optical cavity; $m$ defines the amplitude modulation index; $R$ is the reflective transfer function of the Fabry-Perot cavity. This term vanishes when the laser RAM is nulled.

From literature we found two explanations of the RAM. The first comes from interferences between multiple reflections inside the crystal [11], [12] and the second from polarization misalignment between the front polarizer and the fast axis of the EOM crytal [9]-[11].

\section{EXPERIMENTS AND RESULTS}

For the reduction of RAM, it's useful to use active methods because they are more efficient than passive one [10]-[12]. Regarding the polarization generating RAM, a reduction was made by placing two polarizers before and after the modulator. This made it possible to adjust the polarization of the beam at the input and output of the EOM. Regarding the etalon effect, we implemented a correction of the optical path inside the modulator through a control of the temperature of the crystal as described in [11] as well. The RAM suppression system is shown in figure 2. A laser beam passes through various optical components likes Waves plate (quarter and half), polarizing beam splitter and linear polarizer, before entering the EOM. The phase modulated beam is separated by a beam splitting (50/50) and sent to two fast photodiodes (PD1,2). The RF signal from the photodiode 1 (PD1) is used to control the RAM using a digital PID embedded in Redpitaya (RP1,2) [13]. The photodiode 2 (PD2) is used here to monitor the level of RAM in the system. Two spectrum analyzers (SA) are used for view of the RAM signals. One RP (RP1) is used to drive the EOM at $23 \mathrm{MHz}$ frequency while the other one is used for RAM suppression.

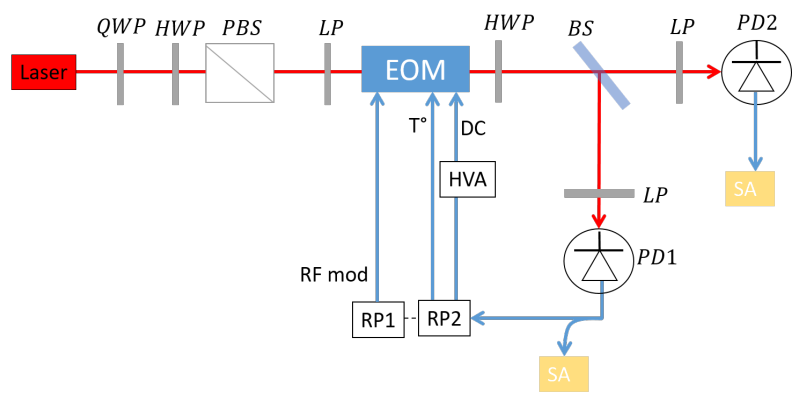

Fig. 2. Experimental setup. P/BS: polarizing/beam splitter; EOM: electrooptic modulator; HVA: high voltage amplifier; LP: linear polarizer; HWP: half wave-plate and QWP:quatter wave-plate; RP: redpitaya; PD: photodiode;

With digital and analog electronic, we have implemented an active control system of the RAM based on in-phase detection and feedback to the temperature and DC voltage ports of the EOM. The level of RAM, characterized by a modulation amplitude index is $2.7 \%$ with a stability of $4.6 \times 10^{-5}$ at $1 \mathrm{~s}$ integration time. This residual amount of RAM is compatible with a fractional frequency stability in the low $10^{-16}$ between $1 \mathrm{~s}$ and $100 \mathrm{~s}$ as we can see on figure 3. It's limited by the relatively low finesse of our cavity [14].

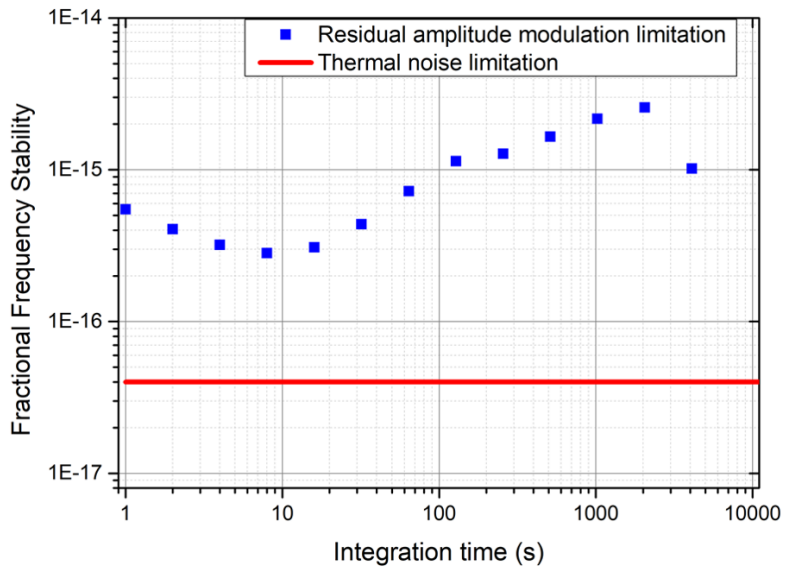

Fig. 3. Experimental measurement of theoretical RAM-induced fractional frequency stability (blue) compared to the thermal noise limit (red) of our Silicium cavity

We can say that, these results are encouraging because improvements can be made in view of the lack of useful resolution of the Redpitaya that we observed, for fast corrections via the DC of the EOM.

\section{CONClusion}

In this work, we have presented the reduction of RAM that we have made. we used digital control to made a compact system. However, we are limited by the resolution of the signal that serves to lock the RAM using the DC voltage output of EOM. It would be important to investigate the temperature sensitivity of the beam splitter placed after the EOM and effect of power fluctuations in the system.

\section{ACKNOWLEDGMENT}

The work has been realized in the frame of the ANR project Equipex Oscillator-Imp. The authors would like to thank the Bourgogne Franche-Comté Regional Council and the FIRSTTF for its support to the Projets d'Investissement d'Avenir for funding.

\section{REFERENCES}

[1] G. K. Campbell, A. D. Ludlow, S. Blatt et al., "The absolute frequency of the 87 Sr optical clock transition," Metrologia, vol. 45, No. 5, (2008), pp. 539-548.

[2] Andrew D. Ludlow, Martin M. Boyd, J. Ye, E. Peik and P. O. Schmidt, "Optical atomic clocks," Reviews of Modern Physics, vol. 87, No. 2, (2015), pp. 637-701

[3] T. Kessler, C. Hagemann, C. Grebing, T. Legero, U. Sterr, F. Riehle, M. J. Martin, L. Chen and J. Ye, "A sub-40-mHz-linewidth laser based on a silicon single-crystal optical cavity," Nature Photonics, vol. 6, No. 10, (2012), pp. 687-692.

[4] B. P. Abbott, R. Abbott, R. Adhikari et al., "the Laser Interferometer Gravitational-Wave Observatory," Rep. Prog. Phys., (2009), vol. 72, No. 7, pp. 076901

[5] K. Döringshoff, I. Ernsting, R-H. Rinkleff, S. Schiller and A. Wicht, "Low-noise, tunable diode laser for ultra-high-resolution spectroscopy," Opt. lett., (2007), vol. 32, pp. 2876-8.

[6] M. Nagel, K. Möhle, K. Döringshoff, S. Schikora, E. V. Kovalchuk and A. Peters, "Ultra-stable cryogenic optical resonators for tests of fundamental physics," Sixth Meeting on CPT and Lorentz Symmetry, (2014), arXiv:1308.5582, pp. 143-148. 
[7] A. Bartels, S. A. Diddams, C. W. Oates, G. Wilpers, J. C. Bergquist, W. H. Oskay and L. Hollberg, "Femtosecond-laser-based synthesis of ultrastable microwave signals from optical frequency references," Opt. Lett., vol. 30, No. 6, (2005), pp. 667-669.

[8] Eric D. Black,"An introduction to Pound-Drever-Hall laser frequency stabilization," American Journal of Physics, Vol. 69, No. 1, (2001), pp. 79.

[9] E. A. Whittaker, M. Gehrtz and G. C. Bjorklund, "Residual amplitude modulation in laser electro-optic phase modulation," J. Opt. Soc. Am. B, vol. 2, No. 8, (1985), pp. 1320-1326.

[10] W. Zhang, M. J. Martin, C. Benko et al., "Reduction of residual amplitude modulation to $1 \times 10^{-6}$ for frequency modulation and laser stabilization,” Opt. Lett., vol. 39, No. 7, (2014), pp. 1980-1983.

[11] Q. Anh Duong, T. Dong Nguyen, T. Tung Vu, M. Higuchi, D. Wei and M. Aketagawa, "Suppression of residual amplitude modulation appeared in commercial electro-optic modulator to improve iodine-frequencystabilized laser diode using frequency modulation spectroscopy," J. Eur. Opt. Soc.-Rapid Publ., vol. 14, No. 14, (2018).

[12] N. C. Wong, and J. L. Hall, "Servo control of amplitude modulation in frequency-modulation spectroscopy: demonstration of shot-noise-limited detection," vol. 2., No. 9, (1985), pp.1527-1533.

[13] B. Marechal, A. Hugeat, G. Goavec-Mérou, G. Cabodevila, J. Millo, C. Lacroûte and P-Y. Bourgeois, "Digital Implementation of Various Locking Schemes of Ultrastable Photonics Systems," International Frequency Control Symposium (IFCS), Olympic Valley, CA, USA, (2018), pp. 1-4.

[14] B. Marechal, S. F. Tetsing Talla, J. Millo, C. Rocher, P-Y. Bourgeois, G. Goavec-Merou, C. Lacroute, E. Rubiola and Y. Kersale, "Development of a Cryogenic Silicon Cavity Stabilized Laser," International Frequency Control Symposium (IFCS), Olympic Valley, CA, USA, (2018), pp. 1-2. 